rev.relac.int.estrateg.segur.13(2):215-243,2018

\title{
La política religiosa del gobierno de Narendra Modi. Pasado y presente del Bharatiya Janata Party en torno a los conflictos interreligiosos en India*
}

\author{
Clarisa Giaccaglia** \\ María Noel Dussort***
}

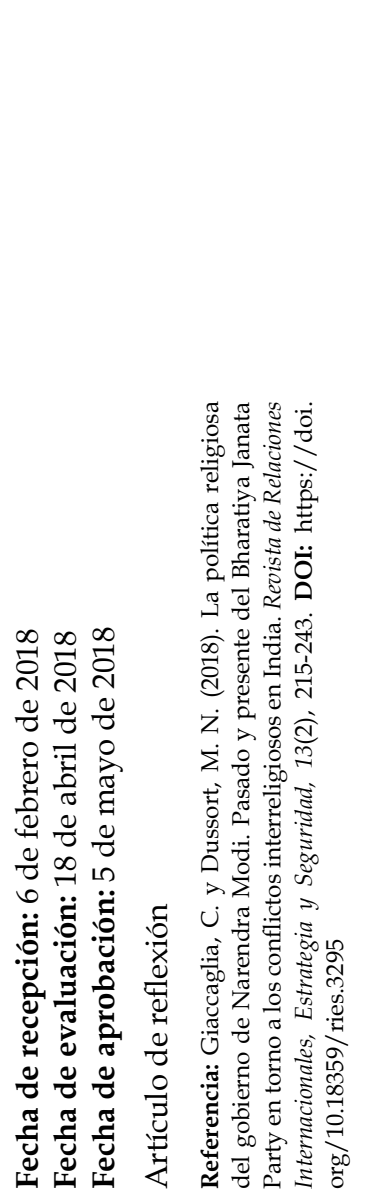

\section{Resumen}

En las elecciones nacionales de 2014, el Bharatiya Janata Party, encabezado por Narendra Modi, logró formar gobierno por primera vez en la historia de India, sin contar con el apoyo del partido del Congreso Nacional Indio. Durante casi cuatro años de mandato, el nuevo gobierno parece haber sostenido una línea política de continuidad con las metas nacionales tradicionales, aunque la política religiosa aparece como una excepción. El artículo tiene por objetivo identificar la manera en que el ideario político y religioso de

* Este artículo se enmarca en el Proyecto de Investigación Plurianual, denominado "El rol de los poderes emergentes en el actual sistema internacional. Desafíos para la inserción de Argentina (2003-2015)", perteneciente al Consejo de Investigaciones Científicas y Técnicas (Conicet).

** Integrante del Consejo de Investigaciones Científicas y Técnicas (Conicet), Argentina. Instituto de Investigaciones, perteneciente a la Facultad de Ciencia Política y Relaciones Internacionales de la Universidad Nacional de Rosario, en Argentina. Correo: clagiaccaglia@yahoo.com.ar

*** Becaria doctoral del Consejo de Investigaciones Científicas y Técnicas (Conicet), Argentina. Instituto de Investigaciones, perteneciente a la Facultad de Ciencia Política y Relaciones Internacionales de la Universidad Nacional de Rosario, en Argentina. Correo: maria. dussort@fcpolit.unr.edu.ar 
los hacedores públicos más relevantes del país ha influido e influye en la conformación de la política religiosa en India. Para tal efecto, se adopta una metodología cualitativa con un enfoque hermenéutico y descriptivo. Consecuentemente, se consideran las distintas perspectivas de los padres fundadores del país, de sus principales académicos y de sus hacedores políticos más relevantes. La conclusión del trabajo apunta a que la política religiosa desarrollada por el gobierno de Modi ha reforzado la discusión sobre la identidad de India con base en dos posturas contrapuestas: una India laica y sincrética o una India basada en la supremacía del hinduismo. Dicha controversia adquiere especial relevancia dadas las repercusiones políticas y de seguridad que este tema podría provocar tanto a nivel nacional como regional.

Palabras clave: Bharatiya Janata Party; hinduismo; India; Narendra Modi; partido del Congreso Nacional Indio; política; religión.

\title{
The Religious Policy of Narendra Modi's Government. Past and Present of the Bharatiya Janata Party Regarding Interreligious Conflicts in India
}

\begin{abstract}
In the 2014 national elections, the Bharatiya Janata Party lead by Narendra Modi could form government for the first time in the history of India without the Indian National Congress' support. For four years of administration, the new government seems to have maintained certain continuity with traditional national goals, although religious policy could be an exception. The aim of this article is to identify how political and religious ideas of politicians have influenced the religious policy in India. For this purpose, we apply a qualitative methodology with a hermeneutic, descriptive approach. Accordingly, we consider the different perspectives of India's founding fathers, main scholars and important politicians. It is concluded that the religious policy developed by Modi's government has reinforced the discussion over India's identity based on two opposing stands: a syncretic and secular India or a Hinduism supremacy-based country. Such controversy becomes more relevant given the political and security repercussions it may have regionally and nationally.
\end{abstract}

Keywords: Bharatiya Janata Party; Hinduism; India; Indian National Congress; Narendra Modi; politics; religion. 


\section{A política religiosa do governo de Narendra Modi. Passado e presente do Bharatiya Janata Party em torno aos conflitos inter-religiosos na Índia}

\section{Resumo}

Nas eleições nacionais de 2014, o Bharatiya Janata Party, encabeçado por Narendra Modi, conseguiu formar governo pela primeira vez na história da Índia, sem contar com o apoio do partido do Congresso Nacional Indiano. Durante quase quatro anos de mandato, o novo governo parece haver mantido uma linha política de continuidade com as metas nacionais tradicionais, ainda que a política religiosa aparece como uma exceção. $\mathrm{O}$ artigo tem por objetivo identificar a maneira em que o ideário político e religioso dos fazedores públicos mais relevantes do país tem influído e influi na conformação da política religiosa na Índia. Para tal efeito, se adopta uma metodologia qualitativa com um foco hermenêutico e descritivo. Consequentemente, se consideram as diferentes perspectivas dos padres fundadores do país, de seus principais acadêmicos e de seus fazedores políticos mais relevantes. A conclusão do trabalho indica que a política religiosa desenvolvida pelo governo de Modi tem reforçado a discussão sobre a identidade da Índia com base em duas posturas contrapostas: uma Índia leiga e sincrética ou uma Índia baseada na supremacia do hinduísmo. Tal controvérsia adquire especial relevância dadas as repercussões políticas e de segurança que este tema poderia provocar tanto a nível nacional quanto regional.

Palavras-chave: Bharatiya Janata Party; hinduísmo; Índia; Narendra Modi; partido do Congresso Nacional Indiano; política; religião.

\section{Introducción}

Históricamente India ha sido identificada como un calidoscopio de etnias, religiones, filosofías, lenguas y dialectos, así como de culturas, rituales, arquitecturas y zonas geográficas. En este sentido, el país puede ser definido como la multiplicidad dentro de la unidad. Dicho principio fue también uno de los ejes rectores al momento de la independencia de India.
En efecto, los padres fundadores consideraron que la presencia de diferentes religiones en el territorio no implicaba, a priori, la existencia de naciones distintas. En consecuencia, una concepción laica del Estado y la laicización de la sociedad permitirían proteger las peculiaridades e insertarse en el proceso de construcción del incipiente país. Jawaharlal Nehru, en particular, excluía la idea de que hubiese un papel para la religión en la vida política de un Estado moderno. 
En términos de D’Orazi, "es inútil especular si este nada fácil compromiso nacía de la convicción de que era realmente posible mantener separados los dos ámbitos, el político y el religioso, o más bien de una prudente reticencia a afrontar una batalla sin esperanzas" (2003, p. 232).

Cabe recordar además, que desde un punto de vista jurídico, el derecho indio -finalmente instaurado al constituirse el Estado independiente, como producto de la prolongada convivencia de varias culturas - fue la comparecencia en un único cuerpo de dos tipos de ordenamiento jurídico: uno laico estatal, de impronta occidental, el cual da orden y administra la vida política y económica del país, y otro personal y familiar, que contempla las tradiciones religiosas y culturales fundamentalmente de los hinduistas y musulmanes, pero también de las restantes religiones de menor presencia numérica'.

En este contexto, resulta importante comprender que, pese a que el laicismo y la libertad religiosa fueron larga- mente discutidos durante la Asamblea Constituyente de 1949, solo en 1976 el gobierno de Indira Gandhi introdujo el adjetivo 'laica' (entre socialista y democrática) en el preámbulo de la Constitución nacional, lo cual identificaba los grandes principios de la soberanía republicana.

Lo cierto es que, aun teniendo en cuenta las sensibilidades que el tema conlleva, el partido del Congreso Nacional Indio (INC, su sigla en inglés), a lo largo de los años y de sus distintos mandatos de gobierno, se distinguió por defender una filosofía laica, sincrética e interclasista con la que se buscó identificar al conjunto del país.

No obstante, durante los primeros años de la década del noventa, India vivió momentos de extrema inestabilidad interna, lo cual se vio reflejado en una oleada sin precedentes de enfrentamientos religiosos entre hindúes y musulmanes. En este contexto, el Bharatiya Janata Party (BJP), partido de carácter conservador, nacionalista y tradicional, adversario del INC, COmenzó a adquirir protagonismo. Por

1 En este sentido, cabe señalar que la Constitución nacional de 1950, en su artículo 372, dispuso la conservación del orden jurídico anterior, o sea, el de todas las leyes promulgadas precedentemente, las cuales, bajo aclaración resolutoria de la Suprema Corte de la India de 1954, no podían ser incompatibles en su contenido con la propia Constitución. Derivado de lo anterior, a través de la British Statutes (Application to India) Repeal Act de 1960, se inventarió un número de ciento cincuenta leyes vigentes anteriores al orden constitucional de 1950, lo cual representa una parte muy considerable del nuevo orden legal establecido. Entre estas leyes se destacan: Ley de Matrimonio Hindú de 1955, que es una actualización de la ley inmediatamente anterior a la Constitución y que regula los derechos personales de los creyentes hinduistas, budistas, jainistas y sijhs. También, Ley de Sucesión Hindú de 1956, Ley Parsi de 1988 con su antecedente inmediato en 1936, Ley familiar cristiana de 2001, que modifica la Ley de 1872, Ley Especial de Matrimonios para los creyentes musulmanes de 1954 (Shariat), que modificó la ley de 1937; Ley Personal Musulmana de 1937, Ley Musulmana de Divorcio de 1939, entre otras (Oropeza, 2015). 
un lado, el por entonces líder del BJP, Lal Advani, encabezó en 1990 una marcha sagrada que atravesó todo el territorio del país y llegó hasta Ayodhya, finalizando en sangrientos episodios. Por otro, A. B. Vajpayee, también perteneciente al BJP, logró acceder al poder nacional (1996-1998) instaurando por primera vez un gobierno de derecha que desarrolló una política dura, nacionalista y a favor de las pruebas nucleares. Se trató, sin embargo, de un gobierno de coalición que no logró sobrevivir por lo que, en poco tiempo, volvió a gobernar el partido del Congreso.

En las últimas elecciones nacionales de 2014, el BJP encabezó la llamada Alianza Democrática Nacional (NDA, su sigla en inglés), integrada por nueve agrupaciones, llevando como principal candidato a Narendra Modi. Con 64 años de edad, Modi se mostró carismático, elocuente y con profundas creencias religiosas. En definitiva y por primera vez en la historia de India, el partido de Modi logró formar gobierno sin contar con el apoyo del partido del Congreso.

Durante sus casi cuatro años de mandato, ya sea por convicción o por simple oportunismo político, el nuevo gobierno parece haber incorporado nuevos métodos o medios, pero sosteniendo una línea política de continuidad con las metas nacionales tradicionales ${ }^{2}$. La implementación de innovadores y mediáticos planes podría tener el objetivo de mantener el apoyo electoral de los sectores rurales que aún siguen representando una abrumadora mayoría del país y que constituyen la puerta de ingreso para un ansiado segundo mandato en 2019. No obstante, la política religiosa aparece como una excepción.

De este modo, surgen algunos interrogantes: ¿qué tipo de política sostiene Modi frente a la cuestión religiosa? ¿Qué antecedentes tiene el BJP en la dinámica política-religión? ¿La llegada del nuevo gobierno ha agravado los enfrentamientos interreligiosos, en especial, entre hindúes y musulmanes? ¿Qué se persigue a través de la llamada "diplomacia del yoga"? ¿Cuáles son las consecuencias de las políticas domésticas que involucran cuestiones religiosas a nivel regional?

El presente artículo realiza una breve revisión histórica sobre la relación entre política y religión desde el momento en que la India se independizó, con

2 Por metas nacionales tradicionales se entiende todas aquellas políticas que se iniciaron a partir del programa de liberalización económica iniciado por el gobierno de India en 1991. Es importante destacar que, más allá de los sucesivos cambios de gobierno, dichas metas iniciales se han sostenido a lo largo del tiempo. Con diferentes estilos y distintas velocidades, las medidas implementadas han sostenido la figura de un Estado fuerte con una función clave en la administración de la economía nacional. A modo de ejemplo, cabe mencionar el sostenimiento de las políticas sociales destinadas a los sectores más vulnerables de la sociedad, pese al reciente cambio de signo partidario en el gobierno nacional. Para un análisis más exhaustivo, se recomienda el artículo titulado "El gobierno de Narendra Modi en India: distintos métodos, iguales metas" (Giaccaglia, 2016). 
el objeto de identificar la manera en que el ideario político y religioso de los hacedores públicos más relevantes del país ha influido e influye en la conformación de la política religiosa en India. Posteriormente, el trabajo se focaliza en el análisis de las acciones de gobierno desplegadas en dicho ámbito a partir de la llegada del BJP al ejecutivo nacional en 2014.

En este sentido, se parte de la idea de que la política religiosa desarrollada por el actual gobierno de Narendra Modi ha reforzado la discusión sobre la identidad de India con base en dos posturas contrapuestas: una India laica y sincrética o una India basada en la supremacía del hinduismo. Dicha controversia no debe ser considerada un dato menor, teniendo en cuenta las repercusiones políticas que este tema podría provocar, dado el alto nivel de sensibilidad inherente a la cuestión, tanto a nivel nacional como regional. Como consecuencia, un manejo poco prudente del tema por parte de Modi podría poner en riesgo uno de los pilares fundamentales que garantizan la estabilidad de la democracia más grande del mundo.

Desde un punto de vista teórico, el artículo busca identificar el modo en que ciertos elementos de política interna tal como la religión pueden incidir en la política exterior de un determinado país, considerando especialmente, en este caso, tanto las posibles repercusiones en la esfera de influencia regional más próxima, esto es, el sur de Asia, así como las implicancias para la imagen internacional de India en cuanto poder emergente del siglo XXI. De este modo, la investigación se enmarca en los estudios de Relaciones Internacionales abocados a la creciente vinculación entre los planos interno y externo (Rosenau, 1973), los juegos de "doble nivel" (Putnam, 1996) o la "diplomacia de doble filo" (Osorno, 1995).

A los fines del presente trabajo, es importante señalar que por política religiosa se entiende el conjunto de tradiciones, concepciones filosóficas e ideológicas, así como el tratamiento jurídico y político que los movimientos religiosos han recibido por parte de las distintas fuerzas políticas participantes del proceso democrático nacional. Dicha política religiosa se ha puesto de manifiesto por medio de distintas prácticas discursivas y acciones gubernamentales que se han desarrollado en el devenir de India como país independiente. En este sentido, se abordan el impacto que la religión ha tenido sobre la política y, al mismo tiempo, el modo en que la política ha incidido sobre la religión, en una relación de mutua influencia.

Cabe aclarar que se parte de una metodología cualitativa, a través de la aplicación de un enfoque hermenéutico y descriptivo, tomando en consideración las distintas perspectivas de los padres fundadores del país, de sus principales académicos y de sus hacedores políticos más relevantes. Por padres fundadores se hace especial 
referencia a Mahatma Gandhi y Jawaharlal $\mathrm{Nehru}^{3}$, actores políticos decisivos al momento de la descolonización del país, si bien tras el fallecimiento del primero se terminará proyectando ampliamente una distintiva concepción nehruviana en la política nacional (Heine, 2013; D'Orazi, 2003). Entre los principales académicos que se han dedicado a un análisis pormenorizado de la vinculación entre política y religión en India, se han tenido en cuenta particularmente los estudios realizados por Amartya Sen, Shashi Tharoor, Thomas Blom Hansen, Christophe Jaffrelot, Romila Thapar y Peter van der Veer. Por último, y en relación a los hacedores políticos más relevantes, se tomaron en consideración los sucesivos primeros ministros del país, así como los principales referentes de la oposición política.

En cuanto a los recursos de investigación abordados en este trabajo, se recurre tanto a fuentes primarias como secundarias. Entre las primarias se cuentan fuentes tales como: 1) discursos políticos de los principales líderes gubernamentales, así como diferentes manifiestos de las fuerzas partidarias gobernantes y de la oposición y 2) documentos jurídicos como la Government of India Act de 1935 del periodo colonial, la Constitución Nacional de 1950 (con sus posteriores enmiendas) y distintas leyes emanadas durante la etapa democrática. Del mismo modo, se utilizaron fuentes secundarias como la literatura especializada, considerando tanto autores de origen indio como occidentales abocados al seguimiento del tema.

El trabajo se divide en dos partes. En primer lugar, se realiza un breve acercamiento a la política religiosa desarrollada por el partido del Congreso a lo largo de sus años de gobierno y a los desafíos que aquel debió afrontar principalmente desde que el hinduismo comenzó a politizarse. En segundo lugar, se hace referencia a los sucesos y acciones gubernamentales acontecidas en el ámbito interno a partir de la llegada de Modi, así como sus repercusiones en la esfera regional.

\section{Consideraciones sobre la cuestión religiosa en el periodo posindependentista}

De acuerdo con el último censo nacional de 2011, el hinduismo es la religión mayoritaria comprendiendo el $80,5 \%$ del total de la población, es decir, alrededor de unos 827 millones de habitantes de una población total de más de 1.200 millones. Por su parte, la religión musulmana reúne la segunda mayor cantidad de fieles, contabilizando el $13 \%$ del total de la población, unos 138 millones, aproximadamente. En tanto país multiconfesional, otras religiones como el cristianismo, el sijismo, el jainismo, el budismo o el zoroastrismo también son de relevancia a nivel numérico pero siguen siendo

3 Como otros de los padres fundadores, cabe destacar también a B. R. Ambedkar y Sardar Patel, quienes no han sido especialmente considerados en este trabajo (véanse Heine, 2013; D'Orazi, 2003). 
marginales en comparación con las dos primeras (Ministry of Home Affairs of India, Census, 2011) ${ }^{4}$.

La conformación religiosa que presenta el país es producto de siglos de penetración cultural a través de múltiples canales (migraciones, invasiones, lazos comerciales, entre otros), dada su posición estratégica en el mapa asiático. Por ello, la capacidad de adaptación a los cambios sufridos a través del tiempo es una característica distintiva de la población india. Según el propio Jawaharlal Nehru cada incursión de elementos extranjeros era un desafío a esta cultura al que se hacía frente con fortuna mediante una nueva síntesis y un proceso de absorción" (1949, p. 99).

No obstante, el proceso de adaptación y convivencia entre grupos religiosos tan heterogéneos ha planteado grandes desafíos para la India contemporánea. El hinduismo y el islamismo merecen especial atención no solo por su condición de religiones mayoritarias, sino además por la historia de conflictos mutuos en la conformación de la India como Estado-nación, los cuales han estado presentes a lo largo de su vida independiente.

En consecuencia, para poder adentrarse en el tratamiento de la religión durante la fase posindependentista, debe hacerse referencia a los años inmediatamente anteriores debido a que marcaron la dinámica de los líderes del partido del Congreso desde 1947 en adelante.

Gran Bretaña, al consolidar su poder en el subcontinente indio en la segunda mitad del siglo XIX, inició un proyecto estatal masivo para enumerar, clasificar y, por tanto, controlar poco más de 250 millones de indios. En este proyecto hubo dos elementos de particular importancia: la acumulación de datos sobre castas y la división de la población en comunidades religiosas. Al respecto, importa considerar que si bien la sociedad de castas existía antes del periodo colonial, los censos (que comenzaron en 1872) hicieron más rígidas aquellas divisiones. Por otra parte, cuando los británicos buscaban aplicar la ley autóctona, se basaban en la simple diferenciación entre la Ley hindú y la Ley de Mahoma (Veer, 1994, p. 19).

Como bien señala Veer:

$[\ldots]$ this is not to say that there was
no division of Hindu and Muslim
communities in the precolonial pe-
riod. There was: the division was
not a colonial invention. But to
count these communities and to
have leaders represent them was
a colonial novelty, and it was fun-
damental to the emergence of reli-

4 Sumando la cantidad de fieles que reúne cada una de estas religiones, no se alcanza a la mitad de musulmanes que se cuentan en el país. 
gious nationalism. (Veer, 1994, pp. $19-20)^{5}$

Los censos trajeron como resultado la división de la sociedad en unidades infinitesimales, con lo cual se creó una abrumadora mayoría hindú junto a varias minorías, siendo la musulmana la más significativa. Las élites políticas trataron tanto de agrandar las comunidades que representaban, como de definir las fronteras entre una y otra de manera más clara. En esta nueva configuración, el número y el comportamiento electoral de la población musulmana se volvieron nuevos hechos de representación política. El Movimiento Pakistán se conformó precisamente debido a la idea de que los musulmanes no podían vivir seguros y con dignidad en un lugar donde no conformaran la mayoría (Veer, 1994, pp. 26-27).

En 1885 se fundó el partido del Congreso como producto de los movimientos nacionalistas de la segunda mitad del siglo XIX. Hacia 1920, Mahatma Gandhi se erigió en el líder de dicho partido y lo convirtió en instrumento de una nueva solidaridad nacional para defender la independencia. Avanzada la lucha contra el colonialismo, Gandhi expresó públicamente su perspectiva sobre las divisiones religiosas:

In the democracy which I have envisaged, a democracy established by nonviolence, there will be equal freedom for all. Everybody will be his own master. It is to join a struggle for such democracy that I invite you today. Once you realize this you will forget the differences between the Hindus and Muslims, and think of yourselves as Indians only, engaged in the common struggle for independence. (Gandhi, 1942) ${ }^{6}$

Para acallar las demandas del partido del Congreso, en 1935 fue sancionada una nueva Constitución por el parlamento británico llamada Government of India Act, la cual constituyó un avance en el proceso hacia la emancipación, aunque ignoraba el tratamiento del problema religioso existente entre hindúes y musulmanes, cada vez más acuciante (Meile, 1962, p. 113). A modo de ejemplo, puede observarse que cuando en dicho documento se alude a la composición de la Asamblea Federal, se distingue entre miembros europeos, anglo-indios, indios-cristianos y, finalmente, miembros de las distintas castas, sin que se haga men-

5 "Esto no es para decir que no había división entre las comunidades hindúes y musulmanas en el período precolonial. La había, pero la división no fue una invención colonial. Aunque censarlas y contar con líderes que las representen fue una novedad colonial, fundamental para la emergencia del nacionalismo religioso" (traducción y cursiva de las autoras).

6 "En la democracia que he imaginado, una democracia establecida por la no violencia, habrá igual libertad para todos. Cada quien será su propio maestro. Esto es para unirse por la lucha por esa democracia que hoy los invito. Una vez que se den cuenta de esto, ustedes olvidarán las diferencias entre los hindúes y los musulmanes y se pensarán como indios solamente, comprometidos en la lucha común por la independencia" (traducción de las autoras). 
ción alguna a otras religiones, particularmente al islam (parte XIV, cláusula primera, art. 26, p. 217).

En las elecciones de 1937, el partido del Congreso ganó el control en ocho de las once provincias existentes en ese momento. Dicho triunfo agudizó los temores de la minoría islámica del país de ser marginada. En efecto, la Liga Musulmana de la India, constituida en 1906, pugnaba desde 1933 para que se erigiera un Estado musulmán autónomo que llevaría el nombre de Pakistán y abarcaría las provincias del noroeste, donde la mayor parte de la población pertenecía a esta religión. Al frente de la Liga estaba Mohammed Ali Jinnah como el más importante portavoz del separatismo.

Como se indicó anteriormente, al interior del partido del Congreso había dos personalidades importantes, que luego pasarían a ser consideradas como los padres fundadores de la India contemporánea: Mahatma Gandhi y Jawaharlal Nehru. Para el primero, la partición del territorio era inaceptable. Nehru, por su parte, no simpatizaba con la idea promovida por la Liga Musulmana pero estaba dispuesto a aceptarla para lograr la independencia (Amikar, 2001, citado en Borella, 2009).

Esta segunda posibilidad fue efectivamente el destino al que se arribó. La constitución territorial actual de India se debe fundamentalmente a la división entre musulmanes e hindúes, producto, por un lado, de la partición del antiguo imperio y la consecuente con- formación de la República de la India, y, por otro, de la República Islámica de Pakistán. La retirada de los británicos de aquellas tierras no se produjo de forma pacífica. Por el contrario, tuvo como resultado el desplazamiento de más de catorce millones de personas de un país al otro según el criterio religioso, causando una ola de violencia que dejó un saldo estimado entre 200 mil y un millón de víctimas.

Desde la perspectiva de Amartya Sen, en los primeros años posteriores a la independencia, el concepto de una identidad india amplia e incluyente conquistó una adhesión generalizada. De hecho,

\section{[...] la determinación de preservar esa identidad de vastos contornos} se fortaleció debido a la profunda sensación de tragedia generada por la partición del subcontinente, y también en virtud del considerable orgullo nacional suscitado por el hecho de que, a pesar de las presiones políticas para lograr un "intercambio de poblaciones", el grueso de los musulmanes de la India independiente decidió permanecer en el país, en vez de trasladarse a Pakistán. (2007, pp. 77-78)

En este sentido, Sen remarca que "esta identidad incluyente, que reconocía y adoptaba la heterogeneidad interna y celebraba la riqueza de la diversidad, estaba acompañada por una inquebrantable negativa a favorecer una comunidad religiosa en desmedro de las demás" (2007, p. 415). 
Además de discrepar respecto de la partición territorial, Gandhi y Nehru también diferían en su forma de pensar la religión como parte de su activismo político. Gandhi tenía una actitud más asertiva con respecto a su identidad hindú, aunque se oponía a dejar que sus idearios religiosos se impusieran a su compromiso general con una identidad india en los asuntos políticos y sociales (Sen, 2007, p. 415). Nehru, en cambio, era agnóstico, tenía ideas socialistas y pretendía que la lucha de la India fuera no solo un movimiento de liberación nacional sino también un movimiento de liberación social.

En palabras de Jaffrelot (2007, pp. 4-5), mientras Gandhi reconocía las identidades religiosas en la esfera pública e incluso concebía la nación como una amalgama de comunidades diferentes, Nehru abogaba por la construcción de una nación basada en individuos, no en grupos. Por consiguiente, la construcción de la nación india solamente podía arraigarse en identidades individuales y seculares.

La posición progresista, laica y modernizadora de Nehru "nunca se situó en términos de oposición a Gandhi, pero con su desaparición ${ }^{7}$ asumió un carácter dominante en los primeros veinte años de vida independiente de la India" (D'Orazi, 2003, p. 20). A raíz de ello, bajo el gobierno del partido del Congreso, India se instituyó como un
Estado laico de facto que favorecía la libertad de culto. Posteriormente, la laicidad se formalizó en la reforma constitucional de 1976, cuando Indira Gandhi decidió introducir el adjetivo "[democracia] laica" en su preámbulo.

El secularismo en India es entendido de forma muy diferente al conocido en el mundo occidental. Existen dos enfoques principales: por un lado, la neutralidad entre las diferentes religiones y, por otro, la prohibición de las asociaciones religiosas en las actividades gubernamentales. Esto da a conocer la necesidad de un Estado neutral entre los diferentes credos debido a la imposibilidad de favorecer por igual la variedad de religiones, no solo por su extensión en número sino por sus extremas disparidades (Sen, 2007; Tharoor, 2007).

Otro ejemplo del compromiso del partido del Congreso con el secularismo fue la abolición de la intocabilidad en la Carta Magna de 1950, la cual se consideró la iniciativa más audaz de ruptura con el pasado. Se trató de un gran gesto simbólico ya que dicha condición nacía de la religión e impregnaba todos los aspectos de la vida social. Textualmente, el artículo 17 reza: "La 'intocabilidad' queda abolida y su práctica, en cualquier forma, prohibida. La aplicación de cualquier incapacidad nacida de la intocabilidad' constituirá un delito penado de

7 El nacimiento de India como país independiente se produjo con la muerte de uno de sus padres fundadores, Mahatma Gandhi quien fue asesinado por un ultranacionalista hindú el 30 de enero de 1948. 
acuerdo con la ley" (Constitución de la India, [1963] 1966).

Posteriormente, en 1955, se sancionó la Reservation Policy, tras la publicación del informe emanado de la Comisión Kalelkar, el cual daba a conocer lo siguiente:

\section{[...] an integrated plan for the re- moval of all causes of backwardness accompanied by ameliorative mea- sures will alone be able to remove this malady inherent in our society. Economic improvement, removal of social inequality, educational ad- vancement and representation of these classes in spheres of power, prestige and authority should form the main features of such a plan. (Government of India, 1955, p. 51) ${ }^{9}$}

Aquella legislación propició la discriminación positiva preservándoles a los ex intocables y los tribales (grupos considerados desfavorecidos) un porcentaje de los puestos en la administración pública y en el sistema educativo ${ }^{10}$ (Cleri y Castillo, 2005, p. 26).

Esta decisión generó resquemores entre las castas altas ya que ponía en peligro la posición social mantenida durante décadas. A su vez, la división entre India y Pakistán, y luego la primera guerra por Cachemira, produjo migraciones forzadas que exaltaron los sentimientos ultranacionalistas y antimusulmanes.

Bajo estas circunstancias, se acrecentó el accionar de una élite india occidentalizada que desde 1920 venía desarrollando una ideología nacionalista basada en una perspectiva reduccionista según la cual la población india es hindú. A pesar de que grandes maestros de la ortodoxia hindú denunciaron el aspecto antitradicional de esta ideología, ya que se oponía a la visión secular pluralista de los hindúes, estas organizaciones ultranacionalistas se fueron consolidando e inspirando numerosos conflictos violentos contra las minorías religiosas, principalmente contra los musulmanes (Accart, 2009, p. 78).

Para Romila Thapar, la comunidad hindú, principalmente la identidad hindú, tomó forma cuando fue necesario sumar adeptos a la causa política. En sus propias palabras: "the need for postulating a Hindu community became a

8 Cabe aclarar que, si bien la edición es de 1963, ésta considera la versión de 1950 y sus posteriores enmiendas, que se encuentran diferenciadas en los anexos.

9 “[...] un plan integral para remover todas las causas del atraso acompañado por medidas de mejora será capaz de remover este problema inherente a nuestra sociedad. El progreso económico, la remoción de la desigualdad social, los avances educativos y de representación de estas clases sociales en las esferas de poder, prestigio y autoridad deberán formar las principales características de este plan" (traducción de las autoras).

10 Es importante mencionar que dicha legislación se mantiene hasta la actualidad, y que fue reformada en 1982 durante el gobierno de Indira Gandhi, aumentando el porcentaje de 10 a 15\% en la discriminación positiva del sector público y a 7,5\% en las instituciones educativas (véase el trabajo de Nityanath y Melkeri, 2017, p. 222). 
requirement for political mobilization in the nineteenth century when representation by religious community became a key to power and where such representation gave access to economic resources" (1989, pp. 229$230)^{11}$. De esta forma, se comenzó a generar un nuevo uso político y social de la religión.

Por tanto, el desarrollo del nacionalismo hindú es un fenómeno moderno que se ha conformado según estrategias de construcción ideológica, a pesar de las características originales del hinduismo como el epígrafe de una diversidad de prácticas (Jaffrelot, 2007, p. 6). El nacionalismo hindú nació en 1920 en Maharashtra, en el contexto de reacción a la movilización panislámica del movimiento Khilafat. Su ideología fue codificada por Savarkar, quien fue el primero que intentó dotar a la nación hindú de una identidad propia, conocida como hindutva, una palabra que él mismo acuñó. De acuerdo con esta perspectiva, los hindúes son los descendientes de los padres védicos, quienes ocuparon el área geográfica del subcontinente indio desde la antigüedad. Además de la religión, la tierra y la raza, Savarkar menciona el lenguaje (el sánscrito y el hindi) como los pilares de la identidad hindú. En suma, la hindutva se basa en el tríptico: "hindu, hindi, Hindustán" (Jaffrelot, 2007, pp. 14-15).
De esta forma, el nacionalismo hindú aparece por primera vez como el resultado de la superposición de una religión, una cultura, un lenguaje y un territorio sagrado, es decir, la receta perfecta para una nacionalidad étnica. Conforme a la hindutva, se asume que los hindúes son el pueblo autóctono de India, mientras que las religiones minoritarias son forasteras $y$, por ende, deben adherirse a la cultura hindú, que es la cultura nacional. En otras palabras, en la esfera privada, las minorías pueden rendir culto a sus dioses y seguir sus rituales, pero en la esfera pública deben pagar lealtad a los símbolos hindúes (Jaffrelot, 2007, p. 15).

La Rashtriya Swayamsevak Sangh (RSS) (Asociación de Voluntarios Nacionales) fue fundada en 1925 y se conformó como el movimiento nacionalista hindú más importante, con la clara intención de propagar la ideología hindutva en India, aunque se mantuvo fuera de la política. El Hindu Mahasabha fue el primer partido político nacionalista hindú, constituido en 1930 bajo el liderazgo de Savarkar. En principio, este partido actuó como fuerza de apoyo del partido del Congreso, hasta cuando el creciente fundamentalismo del primero llevó a este último a alejarse. Más aún, el asesinato de Mahatma Gandhi en manos de un ex miembro de la RSS condujo a Nehru a prohibir las actividades de esta organización.

\footnotetext{
11 "La necesidad de postular a la comunidad hindú la convirtió en un requisito para la movilización política en el siglo diecinueve, cuando la representación de acuerdo a las comunidades religiosas se volvió clave para aspirar al poder y donde tal representación permitió el acceso a recursos económicos" (traducción de las autoras).
} 
No obstante, la RSS no perdió impulso. En 1951 creó el partido político Bharatiya Jana Sangh de bases claramente xenófobas. El Jana Sangh fue solo una de las variadas organizaciones establecidas por la RSS para penetrar en la sociedad india. En 1948, fundó la Akhil Bharatiya Vidyarthi Parishad (Asociación de Estudiantes Indios) para combatir el comunismo en las universidades. En 1955, conformó el sindicato Bharatiya Mazdoor Sangh (Asociación de Trabajadores Indios) En 1964, finalmente, estableció el Vishva Hindu Parishad o VHP (Consejo Mundial de Hindúes) como movimiento responsable de agrupar a los jefes de varias sectas hindúes. Estas y otras organizaciones fueron presentadas como "Sangh Parivar" o la familia de la RSS. Cabe aclarar que muchas de ellas continúan su actividad en el presente.

En 1980, los líderes del Jana Sangh conformaron un nuevo partido polítiCO, el Bharatiya Janata Party (BJP), que se mantuvo fiel a una estrategia moderada, diferente de la ideología original, como forma de ganar aliados en la arena política. Este estilo fue motivo de molestia para todas las organizaciones que conformaban el Sangh Parivar, razón que llevó a la RSS a distanciarse del BJP y a utilizar al VHP para reavivar el activismo político-religioso (Jaffrelot, 2007, pp. 16-20).

A pesar de sus esfuerzos por generar gobierno, el BJP fue durante mucho tiempo una fuerza de coalición, sin tener la fortaleza política para poder lograr gobierno por sus propios medios. No obstante, los acontecimientos de Ayodhya de 1990 lo impulsaron en la escena nacional.

\section{Conflictos religiosos con tintes políticos: desafíos al secularismo defendido por el partido del Congreso}

El secularismo propugnado y defendido por el partido del Congreso desde los fundamentos de la República de la India tuvo sus avatares. El origen de la violencia, como ya se mencionó, radicó en el surgimiento de movimientos fundamentalistas y extremistas que hicieron entrar la política en la religión (Bellino, 2009; Tharoor, 2007). A los fines del presente trabajo, mencionaremos dos de los sucesos más representativos: Ayodhya y Gujarat, debido no solo a la gravedad de los hechos, sino a la particularidad de que el líder actual de India estuvo involucrado en uno de ellos.

La cuestión del templo de Ayodhya proporcionó a los nacionalistas el instrumento para recomponer la comunidad hindú y, al mismo tiempo, ofreció al BJP la posibilidad de volver a entrar en el juego político. Desde mediados del siglo XIX, se fue reforzando entre los hindúes la convicción de que en Ayodhya había existido un templo en honor al dios Rama que los musulmanes habrían destruido, y que era pre- 
ciso reconstruir ${ }^{12}$. En 1949, la cuestión volvió a escena ya que aparecieron las imágenes de Rama y Sita en la mezquita, situación que fue concebida por los hindúes de la localidad como un milagro. Sin embargo, por decisión de Nehru, la mezquita fue cerrada desde 1950, y no fue hasta 1985 que las organizaciones nacionalistas hindúes (principalmente el VHP) comenzaron a reclamar que se reabriera para rendir culto (Jaffrelot, 2007; Veer, 1994; Hansen, 1999). Desde entonces, la localidad se convirtió en escenario de conflicto. Para sorpresa de todos, una orden de la Corte decidió la reapertura de la mezquita en una semana a partir del reclamo iniciado por los nacionalistas hindúes. Según Hansen (1999, p. 150), el cambio de estrategia fue promovido por el partido del Congreso en un intento por ajustarse a la creciente militancia nacional hindú.

En este marco, se deben tener presentes algunos factores. En primer lugar, los musulmanes comenzaron a ganar espacio en la escena política. Desde mediados de los ochenta, el Janata Dal y el Samajwadi surgieron en Uttar Pradesh como los mayores partidos para los musulmanes. Ambos grupos políticos tomaron posiciones firmes promusulmanas contra los reclamos del nacionalismo hindú respecto de la cuestión de la mezquita de Babur. En segundo lugar, el partido del Congreso se encontraba desgastado. La RSS, junto a todo su aparato organizacional, fue cubriendo a nivel local aquellas necesidades básicas insatisfechas y ganando adeptos. Finalmente, dicho activismo político produjo sus frutos en la consolidación del nacionalismo hindú como la segunda fuerza política en India en los años noventa, denominado por algunos autores como la "ola azafrán" (Hansen, 1999).

Ahora bien, en el contexto político de las elecciones parlamentarias de 1984, el partido del Congreso consiguió su mayor victoria, situación que condujo a Vajpayee — presidente del BJP- a cuestionarse su estrategia de postura moderada que había sostenido hasta ese momento. Posteriormente, ante la asunción de Advani como nuevo presidente del BJP, el discurso se volvió más agresivo y se comenzó a acusar al partido del Congreso como "pseudosecularista"13.

12 Para poder contextualizar el problema, es necesario retomar las prosas del poema épico titulado Ramayana, que cuenta que Ayodhya, actual Estado de Uttar Pradesh, fue precisamente el lugar de nacimiento del dios Rama. Los soberanos hindúes que gobernaban la región construyeron un templo en honor a Rama, aunque alrededor del año 1528, bajo la dinastía Mongol, se habría mandado a demoler el templo para construir, en su lugar, una mezquita, conocida desde entonces como "la mezquita de Babur" (D'Orazi, 2003, p. 284).

13 La etiqueta de pseudosecularismo fue una invención del BJP, y le era puesta a cualquier política o medida que no beneficiara a los hindúes como mayoría. Su gran popularidad en los lenguajes locales reflejaba que uno de los efectos profundos de la emancipación de la población a través del proceso democrático había sido la naturalización de los hindúes como la mayoría y como los propietarios de la nación (Hansen, 1999, p. 157). 
Entre los años 1989 y 1993, el enfrentamiento sobre temas de religión y de justicia social tuvo escaladas de máxima conflictividad. En ese momento, la derecha hizo uso de la religión para tratar de unir a la mayoría hindú, mientras que el primer ministro V. P. Singh recurrió a la lógica de castas para neutralizar el llamamiento religioso de los nacionalistas.

En efecto, en 1990 el primer ministro anunció su voluntad de llevar a cabo las propuestas formuladas diez años antes por la Comisión Mandal para profundizar la política de discriminación positiva para las castas más bajas, incrementando el número de plazas reservadas en instituciones educativas y en la administración pública (Veer, 1994). Para el BJP, donde las castas altas constituían el principal pilar del partido, la decisión del primer ministro era inadmisible, ya que ponía en peligro las posiciones de privilegio adquiridas en ambos sectores. Ahora bien, para el partido nacionalista hindú no constituía una decisión fácil oponerse a la discriminación positiva propulsada por el oficialismo, debido a que tal oposición haría titubear el apoyo obtenido entre las castas más bajas, causando la vuelta a la marginalidad política de tiempos anteriores ${ }^{14}$ (D'Orazi, 2003, p. 289).

La respuesta a la decisión de V. P. Singh fue la Rath Yatra (o peregrinación en carro), una marcha sagrada que se efectuó por toda la India en un carro alegórico, exaltando la figura del dios Rama. La peregrinación partió desde Somnath y se extendió hasta Ayodhya, coincidiendo con las ceremonias fijadas para el inicio de la construcción del templo. El recorrido no fue ingenuo, puesto que se marchó por las regiones donde el sentimiento de odio hacia los musulmanes era más intenso, y a su paso se intensificaron los incidentes entre hindúes y musulmanes. Finalmente, el líder del BJP, Lal Krishna Advani, fue encarcelado junto a miles de simpatizantes extremistas. Asimismo, en octubre de 1990, V. P. Singh no autorizó la construcción del templo, lo que motivó que el BJP retirara el apoyo al gobierno nacional (D'Orazi, 2003, pp. 289-290).

Empero, la cuestión de Ayodhya propulsó al BJP como la segunda fuerza política del país después del partido del Congreso. En las elecciones de 1991, se hizo con 119 escaños en el Lok Sabha (Hansen, 1999, p. 166). Más aún, después de la marcha de Ayodhya, hubo dos intentos más de movilización popular por parte del BJP en Cachemira y en Maharastra, aunque estas no tuvieron la ganancia política de la primera.

Después de un periodo de calma, en 1992 el caos volvió a inundar Ayod-

14 Cabe mencionar que el BJP desde fines de 1980 comenzó a ganar gran cantidad de escaños en el Lok Sabha, pasando de ochenta y cinco asientos en 1989 a ciento sesenta y uno en 1996. Respecto a lo anterior, véase el sitio web del BJP. También fue ganando posiciones a nivel provincial, como en Gujarat, Uttar Pradesh. 
hya. Todo comenzó con las iniciativas de Kalyam Singh, jefe de gobierno de Uttar Pradesh, que consistieron en construir un templo hindú sobre el terreno adyacente a la mezquita, para ganar prestigio en ambos bandos religiosos. La ceremonia inaugural fue designada para el 6 de diciembre de 1992, lo cual generó la movilizaron de cientos de kar sevak, jóvenes fanáticos entregados a la causa. El desenlace fue catastrófico: después de una breve aparición de los líderes políticos, la masa de jóvenes perdió el control y comenzó la demolición de la mezquita. Pero los sucesos no se disolvieron allí; muy por el contrario, la violencia se propagó por toda la ciudad y luego por varios Estados del país, la cual dejó un saldo de 1.200 víctimas, siendo Bombay la ciudad que registró los actos más sangrientos (D'Orazi, 2003, pp. 300-302). La violencia también se propagó a Pakistán, Bangladesh y Gran Bretaña, incendiando templos hindúes en represalia por lo acontecido (Veer, 1994).

En segundo lugar, importa dar a conocer los incidentes de Godhra, en el Estado de Gujarat, los cuales tuvieron características similares a los sucesos de Ayodhya. El 27 de febrero de 2002, un tren cargado con 1.700 activistas de la derecha hindú, se detuvo en Godhra, encontrándose de regreso de una peregrinación en Ayodhya. En la estación estalló una pelea entre dichos activistas y los vendedores, en su mayoría musulmanes. El tren terminó incendiado y alrededor de cincuenta y ocho individuos murieron. En una ola de represalias por parte de extremistas hindúes, se emprendió contra los musulmanes una limpieza étnica que terminó con la vida de más de 2.000 personas (Festa, 2009, p. 16).

El desenlace de los acontecimientos aludidos estuvo sujeto más al usufructo que los partidos políticos hicieron de aquellos, que a los sentimientos religiosos presentes en la población india. Para Tharoor (2007), lo sucedido en Ayodhya representó un cambio considerable en los fundamentos sobre los que se concibió el país inmediatamente después de su independencia. En otras palabras, la noción de nacionalismo indio pensado por Nehru rechazaba la religión como determinante de esa nacionalidad.

Para los musulmanes indios, la mezquita destruida de Ayodhya tenía que ver con su lugar en la sociedad india. Durante décadas, los sucesivos gobiernos habían garantizado su seguridad en un Estado secular. Dicho ataque, en cambio, se produjo bajo la inacción de las autoridades subnacionales y la policía.

Es por ello que, después de casi sesenta y siete años ininterrumpidos de gobierno del partido del Congreso, la asunción de Narendra Modi provocó preocupación entre las minorías religiosas. Un acercamiento a la política religiosa de este nuevo gobierno nos permitirá entonces reflexionar sobre si se están cuestionando los basamentos pluralistas de esta nación asiática. 


\section{La llegada de Narendra Modi al poder: controversias frente a la política religiosa}

Desde la campaña electoral de 2014, Narendra Modi debió sortear fuertes críticas vinculadas a su visión política sobre el asunto religioso y a sus posibles medidas en caso de acceder al gobierno nacional. Dicha desconfianza se explicaba tanto por su posición personal frente al tema, como por su accionar en el pasado como funcionario de gobierno en Gujarat.

Como se señaló anteriormente, el partido gobernante (BJP) posee estrechos vínculos con la Rashtriya Swayamsevka Sangh (RSS), organización líder del nacionalismo. En este contexto, debe destacarse que Modi es personalmente un hombre profundamente religioso que profesa el hinduismo y que desde los ocho años es miembro de la RSS, habiendo trabajado activamente en dicha organización.

Durante su gestión en el Estado de Gujarat, ocurrieron los ya mencionados violentos disturbios de 2002 entre extremistas hindúes y musulmanes, que generaron una aguda controversia en torno a la figura de Modi. En dicho episodio, Modi fue acusado de dividir a India en bandos religiosos. Varios procesos judiciales conniventes eximieron de todo tipo de responsabilidad al líder del BJP. Sin embargo, gran parte de la opinión pública del país siguió atribuyéndole por lo menos un grado de complicidad en la masacre, acusándolo de no haber ordenado una adecuada intervención de las fuerzas de seguridad.

Obedeciendo a estas razones, durante la campaña electoral nacional de 2014, la gran mayoría de la comunidad musulmana se opuso a la candidatura de Modi y votó por el partido del Congreso, que todavía sigue siendo valorado como el principal garante de un Estado secular. En contrapartida, Modi contó fuertemente con el apoyo de los nacionalistas hindúes.

Si bien Modi ha intentado apaciguar esta controversia mostrando un perfil moderado, la polémica ha continuado durante sus primeros años de mandato. En este sentido, el BJP ha retomado la propuesta para la construcción de un templo en Ayodhya (Uttar Pradesh) - lugar que, como ya se mencionó, ha sido motivo de muchas batallas entre religiones-, así como la elaboración de un código civil único con el velado objeto de eliminar la actual normativa que rige para minorías como los musulmanes.

Es importante señalar que las mayores críticas hacia el gobierno han provenido de las clases medias, las cuales se han sentido defraudadas por la nueva administración nacional frente a la suba de impuestos. Sumado a ello, no ven con buenos ojos muchos de los acontecimientos sucedidos que, desde su perspectiva, están minando la libertad de expresión en el país.

Los detractores del actual gobierno aseguran que los linchamientos pú- 
blicos por razones religiosas se han incrementado desde el inicio de esta gestión nacional. Asimismo, la comunidad académica universitaria ha denunciado el sistemático deterioro del derecho a la expresión y el ambiente de intolerancia por el que está atravesando la nación. Diversos grupos religiosos, además, se han alarmado por los frecuentes ataques contra iglesias en distintas zonas del país (Deepalakshmi, 2016).

Entre los acontecimientos que más repercusión periodística ha tenido, cabe destacar el suicidio de un docente investigador dalit (intocable) en la Hyderabad Central University y los cargos de sedición contra estudiantes de la Jawaharlal Nehru University. Estos últimos provocaron gran consternación, debido a que dichas medidas eran aplicadas durante el periodo colonial a todo aquel que osara expresar públicamente su opinión en contra de las autoridades británicas. A estos hechos deben sumarse las huelgas de los estudiantes del Film and Television Institute of India (FTII), la decisión de no apoyar el establecimiento de centros fuera del campus de la Universidad Musulmana de Aligarh y el cierre del Ambedkar Periyar Study Circle (APSC) en el marco de los Indian Institutes of Technology en Madras (González, 2016; Deepalakshmi, 2016).

En consecuencia, Modi tuvo que publicar varias declaraciones, reunirse con los líderes religiosos de los grupos minoritarios y enviar una ofrenda simbólica a Ajmer Sharif Dargah, el famo- so santuario sufí del siglo XVII, a fin de serenar los ánimos.

Del mismo modo, a nivel internacional, ha intentado moderar el efecto nocivo de los hechos mencionados y capitalizar el éxito cosechado por el yoga en Occidente, a fin de mostrar al mundo la cara benévola del hinduismo. Dicho objetivo ha sido perseguido a través de la llamada diplomacia del yoga.

La diplomacia del yoga desarrollada por Modi implicó importantes gestiones personales del primer ministro indio ante el secretario general de las Naciones Unidas para el establecimiento de un día internacional del yoga (el 21 de junio), el cual fue finalmente instituido por Naciones Unidas en 2015 (Mukhopadyay, 2015). En su primer aniversario, el día fue conmemorado con multitudinarias clases al aire libre en muchas ciudades del mundo. Asimismo, el gobierno de Modi creó un nuevo Ministerio de Ayurveda, Yoga y Naturopatía, el cual ha logrado gran visibilidad en todos los medios de comunicación.

Cabe recordar que el yoga adquirió fama mundial a partir de la llegada de Maharishi Mahesh Yogi a Estados Unidos, en la década del sesenta, quien popularizó la meditación transcendental. Esta práctica se convirtió en moda en Occidente y tuvo su momento de apogeo con la anunciada visita de The Beatles a la ciudad india de Rishikesh. Hoy en día, el yoga se ha convertido también en un gran negocio. Solo en 
Estados Unidos, es un sector valorado en diez mil millones de dólares anuales. Hay sondeos que indican que casi veinte millones de personas lo practican a diario en ese país (Mukhopadyay, 2015).

Se observa, entonces, que el interés por el yoga es anterior al ascenso de Modi al poder y a la declaración del Día Internacional. La novedad radica en que el gobierno de Modi pretende utilizar el yoga como herramienta política, con vistas a mejorar la imagen internacional del país.

No obstante, a nivel doméstico, el yoga agudizó las discusiones políticas entre el discurso oficial —que lo considera una práctica inofensiva- $-y$ los musulmanes, quienes perciben el yoga como una cuestión religiosa que esconde la pretensión del gobierno de convertirlo en un símbolo de identidad nacional, desconociendo las otras religiones e identidades que coexisten en el país. Por consiguiente, la celebración del primer Día Internacional del Yoga contó con una gran oposición por parte de la población musulmana de India.

Narendra Modi se ha preocupado por acallar las críticas sobre su inclinación hacia un mayor protagonismo del hinduismo en el Estado. El 17 de febrero de 2015, el primer ministro de India aseguró, en un evento en honor a los santos católicos indios, que:

My government will ensure that there is complete freedom of faith and that everyone has the undeniable right to retain or adopt the religion of his or her choice without coercion or undue influence. My government will not allow any religious group, belonging to the majority or the minority, to incite hatred against others, overtly or covertly. (Modi, 2015b) $)^{15}$

Ahora bien, la polémica continúa vigente en virtud de dos acontecimientos. En primer lugar, en 2018 se cumplen veinticinco años de la tragedia de Ayodhya. Fundamentalistas hindúes pretenden llevar a cabo una nueva campaña como en 1992, en pos de la construcción de un templo hindú en el predio de la otrora mezquita musulmana. Dicha situación ha generado renovadas tensiones interconfesionales (Gowen, 2018). En segundo lugar, la cercanía de nuevas elecciones presidenciales en 2019 agudiza el clima de campaña electoral, potenciando el enfrentamiento entre el bloque oficialista y el ahora opositor partido del Congreso, encabezado por Rahul Gandhi. En este contexto debe entenderse el incidente ocurrido con el último representante de la dinastía Nehru, quien en 2017

15 "Mi gobierno asegurará que haya completa libertad de culto y que cada quien tenga el derecho inalienable de retener o adoptar la religión de su elección sin coerción o influencia injustificada. Mi gobierno no permitirá que ningún grupo religioso — perteneciente a la mayoría o la minoría — incite el odio en contra de otros, abiertamente o de manera encubierta" (traducción de las autoras). 
durante la visita a un templo hindú, en Gujarat, fue obligado a registrar su ingreso como "no-hindú". Frente a este hecho, Rahul Gandhi sostuvo que "la religión es un asunto del ámbito privado" (2017). Sumado a esta controversia, activistas de derechos humanos han dado a conocer su preocupación ante la posibilidad de que un segundo mandato del BJP propicie la declaración de India como un Estado hindú (Rediff News, 2017).

En definitiva, las desavenencias en torno a la política religiosa constituyen una temática de discusión permanente, atentando contra el delicado equilibrio interreligioso que caracteriza a India.

\section{La política religiosa de Modi y su impacto en la región}

En India se encuentran las poblaciones suníes y chíes más importantes del mundo, después de Indonesia e Irán, por lo que la paz social interna depende de su feroz resistencia a cualquier alineación exterior que tenga como blanco alguna religión. Este factor explica, por ejemplo, por qué la India está dispuesta a mantener fuertes lazos tanto con Israel como con Irán (Sahni, 2013, p. 112).

Ante los antecedentes que presentó Modi en sus gestiones gubernamen- tales, así como ante el discurso nacionalista hindú utilizado en su campaña electoral, se vaticinaron tensas relaciones con algunos de sus vecinos inmediatos, como por ejemplo Pakistán o Bangladesh. Sin embargo, tal como se adelantó, la política exterior de Modi hasta la fecha ha tenido un alto contenido pragmático. El lanzamiento de políticas puntuales a nivel externo como Neighbourdhood First Policy (Política de Primero el Vecindario) y, luego, el renombramiento de la Look East Policy (Política de Mirada al Este) como Act East Policy (Política de Actuar Hacia el Este) fueron señales de continuidad con el gobierno de Manmohan Singh. En particular, la Política de Primero el Vecindario tenía como objetivo profundizar en la cooperación y la conectividad regional (Modi, 2015a), lo cual se puede constatar en los vínculos que se detallan a continuación.

En relación con Pakistán, contrariamente a lo que muchos esperaban, no se adoptó, en principio, una línea dura $^{16}$. De hecho, Modi invitó al primer ministro paquistaní, Nawaz Sharif, a su toma de posesión, entendiendo que los enfrentamientos intermitentes con el país vecino desviaban su atención de la economía. Posteriormente, en diciembre de 2015, Modi viajó a Pakistán, lo cual no fue anunciado de forma oficial y supuso la primera visita

16 Cabe recordar que India y Pakistán mantienen un conflicto territorial desde sus respectivas independencias, lo cual Ilevó a ambos países a tres guerras: en 1947 y 1965, por la cuestión de Cachemira, y en 1971, una guerra asociada a la independencia de Bangladesh. Finalmente, en 2003 se acordó un alto al fuego que sigue vigente, aunque ambos países se acusan mutuamente de violarlo. 
en más de una década de un primer ministro indio al país vecino (Fontdegloria, 2015).

De esta manera, el gobierno de Modi busca el establecimiento de relaciones pacíficas, en pos de concentrarse en el crecimiento económico del país. Desde la contraparte paquistaní, el primer ministro Sharif también defendió las ventajas de una mayor cooperación económica bilateral (Campos, 2014, p. 5). No obstante, el desarrollo de la relación está fuertemente condicionado por el estallido de atentados por parte de terroristas, que podrían aprovechar este periodo de transición política como una oportunidad para hacer fracasar cualquier tentativa de paz. De hecho, esta situación es la que luego parecería que no podría evitarse a partir de una nueva escalada de violencia desde septiembre de 2016, que ha generado la realización de "ataques quirúrgicos" por parte de India contra terroristas en Cachemira (Olazabal, 2016).

La llegada al poder de Narendra Modi tampoco prometía un futuro alentador para las relaciones con Bangladesh. Las declaraciones realizadas durante la campaña electoral, a través de las cuales se denunciaba la inmigración ilegal proveniente del vecino país, dejaron ver que las relaciones serían tensas. Al respecto, la retórica sobre la cuestión de la inmigración ilegal que permea los lazos entre India y Bangladesh no es nueva. Justamente, el BJP siempre ha realizado una distinción entre los inmigrantes hindúes y los musulmanes, concibiendo a los primeros como refugiados y a los segundos como inmigrantes ilegales (Pattanaik, 2014).

No obstante, el gran logro del primer ministro indio radica en haber conseguido la ratificación del Acuerdo de Fronteras de 1974, así como el Protocolo adicional de 2011, en mayo de 2015, un mes antes de su visita oficial. En efecto, ambos países lograron un acuerdo histórico por el cual se simplificó una frontera de 4.150 kilómetros y se otorgó la posibilidad a más de cincuenta mil personas de tener la ciudadanía del territorio que les rodea. Al desaparecer los enclaves existentes ${ }^{17}$, los residentes tienen dos opciones: quedarse en sus domicilios y adoptar una nueva nacionalidad, o conservar la actual mudándose al Estado del que han formado parte desde hace décadas (Olazabal, 2016).

La clave de este resultado histórico fue el hecho particular de que el BJP tenía mayoría a nivel parlamentario. No obstante, es interesante recalcar que el partido nacionalista hindú se había interpuesto reiteradamente a la ratificación del acuerdo mientras fue la principal fuerza de oposición.

17 En el intercambio de soberanía, India renuncia a ciento once enclaves de novecientos diecisiete hectáreas cercadas por suelo bangladesí en las que viven 37.370 personas, mientras que Bangladesh cede cincuenta y un islas de 1.123 hectáreas con 14.200 habitantes (Olaabal, 2016). 
En el caso de Afganistán, y frente al retiro de las fuerzas de la OTAN de dicho país ${ }^{18}$, se debía decidir la naturaleza y el modo de la presencia india en este Estado. Kabul se ha convertido en el segundo destino de la cooperación internacional india después de Nepal, por su compromiso en asistir a la reconstrucción del país. A nivel mundial, India es el sexto mayor socio, habiendo aportado dos mil millones de dólares desde 2001 (Haidari, 2015).

Este compromiso con el vecino país se debe a la lucha contra focos terroristas, muchos de ellos provenientes de Pakistán. Nueva Delhi está particularmente interesada en la estabilidad de Afganistán ya que otra guerra civil tendría serias consecuencias en la seguridad de India. También, ese interés obedece a la inestabilidad que la guerra generaría en la región y a la posibilidad de un incremento de atentados de radicales islamistas, considerando la delicada situación de la región de Cachemira.

Si bien las primeras acciones de Modi referidas a la Cooperación Sur Sur (CSS) denotan que India continuará ubicando a sus vecinos países en primer lugar, la partida presupuestaria aprobada para el área de la Cooperación al Desarrollo por el gobierno anterior en el periodo 2014-2015, fue considerablemente disminuida en 2015-2016. Esta situación podría poner en duda el compromiso de India con Afganistán, así como con Nepal y Bután, los tres principales receptores de proyectos de cooperación ${ }^{19}$, particularmente frente al avance de China en el sur de Asia.

En definitiva, con dos años y medio en el poder, se observa que la política regional de Modi ha sido poco influenciada por el fundamentalismo de la hindutva. Las disputas pendientes de resolución son producto de antiguas antinomias en el proceso de consolidación del Estado-nación, las cuales se mezclan con amenazas a la seguridad nacional propias de la era posmoderna.

\section{Conclusión}

Tal como se ha observado en el presente análisis, a lo largo de la historia han convivido diferentes religiones en el territorio indio. No obstante, hacia fines del siglo XIX y principios del XX, el colonialismo británico fue una de las causas que provocó la emergencia de un nacionalismo religioso, que tuvo su punto cumbre en la partición del territorio y la conformación de dos estados distintos de acuerdo con las dos religiones mayoritarias: hinduismo e islam.

A pesar de que la vida independiente de India se inició bajo la sombra de la división y la masacre, los padres fundadores se esforzaron en pos de

18 Las tropas de la OTAN se retiraron de Afganistán en diciembre de 2014.

19 Para profundizar en la temática de la Cooperación Sur-Sur de India, se recomienda consultar el trabajo de María Noel Dussort (2016), titulado “¿Donante emergente o socio en el desarrollo? Un análisis de la Cooperación Sur-Sur de India en el siglo Xxı". 
que un concepto de identidad india amplia e incluyente, tuviese una adhesión generalizada. Por ello, tanto en lo discursivo como en la práctica, los sucesivos gobiernos del partido del Congreso Nacional Indio defendieron la idea basal de secularismo, es decir, la separación del Estado de cualquier orden religioso específico. Dicha concepción debe edificarse en un Estado equidistante de todas las religiones, que se niegue a tomar partido y que tenga una actitud neutral hacia ellas.

Esta forma de concebir la esfera pública se erigió como una garantía para gran parte de los musulmanes residentes en el país, quienes luego de la partición del territorio decidieron permanecer en India en vez de trasladarse a Pakistán. Sin embargo, el desgaste propio de la continuidad en el poder del partido del Congreso (principalmente de la familia Nehru), sumado a la falta de respuesta a problemáticas sociales y económicas urgentes en regiones vulnerables del país, provocaron el surgimiento y afianzamiento del BJP a nivel nacional, a partir de los años noventa. Es decir, si bien los conflictos interreligiosos nunca dejaron de estar presentes, las tensiones se agudizaron desde la última década del siglo XX.

Aunque el BJP fue fundado sobre el ideario del nacionalismo hindú, no fue sino el uso oportunista por parte de sus representantes, quienes resucitaron viejas antinomias invocando la identidad hindú solo para sumar adeptos a la causa política. Este utilitarismo religioso tuvo su máxima expresión en los acontecimientos de Ayodhya y Gujarat, funcionando de trampolín a la "ola azafrán". Asimismo, es interesante el hecho de que el BJP utilizó manifestaciones religiosas - que derivaron en enfrentamientos violentos entre hindúes y musulmanes - para apaciguar medidas tomadas por el partido del Congreso que los perjudicaba en sus posiciones de poder.

Frente a estos antecedentes del BJP, el análisis realizado en el presente artículo -retomando el interrogante inicialpone de manifiesto que la política religiosa desarrollada por el actual gobierno de Narendra Modi ha reforzado la discusión sobre la identidad de India con base en dos posturas contrapuestas: una India laica y sincrética o una India basada en la supremacía del hinduismo.

En efecto, la idea tradicionalmente sostenida por el BJP, sobre todo por la RSS, se basa en que la India es, en esencia, un "país hindú", razón por la que sería un gran error, desde un punto de vista estadístico y cultural, tratar el hinduismo como una más entre las distintas religiones presentes en el país. Según esta perspectiva, el hinduismo hace que la India sea lo que es, y la apelación al secularismo, con su insistencia en un tratamiento simétrico de las diferentes religiones, se convierte en un desatino político.

Pese a ello, desde su llegada al poder, el BJP ha actuado con un perfil crecientemente pragmático a través del cual mantiene el discurso tradicional, sobre todo a nivel doméstico, aunque mo- 
derando su conducta en los hechos, especialmente con sus países vecinos. De este modo, se retoma lo sostenido en la introducción al comprobarse que las acciones desplegadas a nivel doméstico conllevan implicancias en el ámbito de proyección externa. Así, la religión no se ha mantenido exenta de esta tendencia.

En este sentido, la administración del primer ministro parece llevar la cuestión religiosa de forma más prudente a nivel doméstico, con el claro objetivo de seguir ganando fuerza política para un segundo mandato en 2019. A su vez, sus acciones se vinculan más con una forma de proyectar a India a nivel global —a través de ciertos símbolos culturales propios-, que como una forma de imposición del hinduismo en la propia sociedad india. Ejemplo de ello fue la institución del Día Internacional de Yoga por las Naciones Unidas.

En cuanto a su entorno regional, cabe mencionar que las relaciones con Pakistán se mantienen al margen de las cuestiones religiosas. De esta manera, los últimos enfrentamientos deben ser leídos sobre la consigna de la seguridad nacional, a pesar de que, ciertamente, muchos de los grupos terroristas asentados en territorio pakistaní justifiquen sus actos en creencias religiosas.

Ahora bien, el debate permanece abierto. Siguiendo las palabras de Amartya Sen (2007), la argumentación sobre una India "hindú" se basa en una lectura sobre la historia y la cultura indias muy superficial, pues:
[...] la herencia cultural de la India contemporánea combina influencias islámicas con tradiciones hindúes y otras, y los resultados de la interacción entre miembros de diferentes comunidades religiosas puede verse en toda su plenitud en la literatura, la música, la pintura, la arquitectura y muchos otros campos. (pp. 374-375)

En rigor, sintetiza finalmente el premio Nobel, "hasta la naturaleza de las creencias y prácticas religiosas hindúes ha sufrido una influencia sustancial de las ideas y valores islámicos".

Por tanto, la identidad no debe ser "una cuestión de historia o descubrimiento sino también de razonamiento, en el cual debe intervenir una elección" (Sen, 2007, p. 416). En este sentido, volvemos entonces a preguntamos: ¿qué identidad quiere la India para sí misma? ¿De qué manera sus gobernantes conducirán la cuestión religiosa para una nación del siglo XXI, en un contexto internacional con fuertes enfrentamientos, con un terrorismo que se excusa bajo un discurso religioso pero que solo encierra intolerancia? En un mundo que, en tiempos recientes, parece abogar por la construcción de muros, desconociendo la diversidad cultural que hoy en día conforma a la mayor parte de los estados, producto de un planeta crecientemente globalizado, resta permanecer expectantes, a fin de poder discernir cuál será la posición de la democracia más grande del mundo. 


\section{Referencias}

Accart, X. (2009). El hinduismo, anclado en su territorio. En J. P. Denis y A. Frachon (eds.), El atlas de las religiones (pp. 78-79). Buenos Aires: Capital Intelectual.

Bellino, F. (12 de septiembre de 2009). Puerta que se abre al infinito. En India, entre el cielo y la tierra. Revista $\tilde{N}$.

Bharatiya Janata Party (BJP). (s. f.). History. Recuperado de http://www. bjp.org/about-the-party/history

Borella, G. (2009). India y la democracia: ¿herencia o tradición? La etapa del nehruvianismo (1947-1964). Grupo de Estudios sobre India y el Sudeste Asiático de Rosario, Programa de Relaciones y Cooperación Sur-Sur. Recuperado de http:// www.precsurweb.com.ar/images/ publicaciones/India_Borella.pdf

Campos, R. (11 de junio de 2014). Histórica victoria electoral de Modi abre un nuevo ciclo político en la India. Real Instituto Elcano. Disponible en http://www. realinstitutoelcano.org/wps/portal/ rielcano_es/contenido?WCM GLOBAL_CONTEXT=/elcano/elcano_es/zonas_es/asia-pacifico/ ari28-2014-campos-victoria-electoral-modi-nuevo-ciclo-politico-india

Cleri, P. y Gallo, M. (2005). Historia reciente. En G. Baez (coord.), Argentina-India: un desafío y una oportunidad para la vinculación económica y comercial. Buenos Aires: Cepal.

Constitución de la India, reformada al 1 de marzo de 1963. (1966). Buenos Aires, Argentina: Gráficos Julio Kaufman SRL.

D'Orazi, F. (2003). Historia de la India: de la independencia de 1947 a nuestros días. México, D. F.: Océano.

Deepalakshmi, K. (25 de mayo de 2016). Two years of Modi government: a review. The Hindu. Recuperado de http://www.thehindu.com/ news/national/twoyearsofmodigovernmentareview/article8640603. ece? css = print

Dussort, M. N. (2016) ¿Donante emergente o socio en el desarrollo? Un análisis de la Cooperación Sur-Sur de India en el siglo xxı. En G. Lechini y C. Giaccaglia (eds.), Poderes emergentes y Cooperación Sur-Sur: perspectivas desde el Sur Global (pp. 86-112). Rosario: UNR Editora.

Festa, R. (12 de septiembre de 2009). La violencia de la derecha hinduista. En India, entre el cielo y la tierra. Revista $\tilde{N}$.

Fontdeglòria, X. (25 de diciembre de 2015). India y Pakistán impulsan el deshielo en una reunión sorpresa. El País. Recuperado de http://internacional.elpais.com/ internacional/2015/12/25/actualidad/1451058414_471796.html

Gandhi, M. (8 de agosto de 1942). Quit India Speech. En A. Sharma (comp.), Quit India Speech by Mahatma Gandhi. (1942). Recu- 
perado de https:/es.scribd.com/ document/143583638/Quit-IndiaSpeech-by-Mahatma-Gandhi-1942

Giaccaglia, C. (2016). El gobierno de Narendra Modi en India: distintos métodos, iguales metas. Cuadernos de Política Exterior Argentina, 124(6), 3-25.

González, M. (09 de junio de 2016). A dos años del gobierno de Narendra Modi: intolerancia, desilusión y esperanza. Foreign Affairs Latinoamérica. Recuperado de http:// revistafal.com/a-2-anos-del-gobierno-de-narendra-modi/

Government of India. (1955). Report of the India backward classes Commission. I. Shimla: The Manager Government of India Press.

Gowen, A. (12 de marzo de 2018). India's Hindu right intensifies a religious battle over a demolished mosque. The Washington Post. Recuperado de https://www.washingtonpost.com/world/asia_pacific/ indias-hindu-right-intensifies-a-religious-battle-over-a-demolishedmosque/2018/03/11/7a35de6a-1 70b-11e8-930c-45838ad0d77a story.html? noredirect $=$ on \&utm _ term $=.545571 \mathrm{abc8fa}$

Haidari, M. (16 de septiembre de 2015). India and Afghanistan: A Growing Partnership. The Diplomat. Recuperado de https:/thediplomat.com/2015/09/india-and-afghanistan-a-growing-partnership/

Hansen, T. (1999). The Saffron Wave: Democracy and Hindu Nationalism in Modern India. Princeton, NJ: Princeton University Press.

Heine, J. (2013). La nueva India. Santiago: Penguin.

In 2019 RSS will declare India a Hindu Rashtra. (17 de abril de 2017). Rediff News. Recuperado de http:// www.rediff.com/news/interview/ in-2019-rss-will-declare-india-ahindu-rashtra/20170417.htm

Jaffrelot, C. (ed.). (2007). Hindu Nationalism: $A$ reader. Princeton, NJ: Princeton University Press.

Langa, M. (1 de diciembre de 2017). Religion a private matter: Rahul. The Hindu. Recuperado de http:// www.thehindu.com/todays-paper/ tp-national/religion-a-private-matter-rahul/article21237172.ece

Meile, P. (1962) Historia de la India. Buenos Aires: Eudeba.

Ministry of Home Affairs, Government of India. (2011). 2011 Census Data. Recuperado de http://censusindia. gov.in/2011-Common/CensusData2011.html

Modi, N. (6 de junio de 2015a). PM Narendra Modi's statement during joint press briefing with his Bangladesh counterpart Sheikh Hasina. The Indian Express. Recuperado de http://indianexpress. com/article/india/india-others/ pm-narendra-modis-statement-during-joint-press-briefing-with-bangladesh-pm/

Modi, N. (17 de febrero de 2015b). Will ensure complete freedom of faith: Full text of PM Modi's 
speech at Christian conference. Firstpost. Recuperado de https:// www.firstpost.com/india/will-ensure-complete-freedom-of-faith-fulltext-of-pm-modis-speech-at-christian-conference-2103923.html

Mukhopadyay, N. (15 de agosto de 2015). La diplomacia del yoga. El País. Recuperado de http:// internacional.elpais.com/internacional/2015/08/14/actualidad/1439547511_232032.html

Nehru, J. (1949) El descubrimiento de la india (1889-1964). Buenos Aires: Editorial Sudamericana.

Nityanath, S. y Melkeri, S. P. (2017). Reservation policy in India and development of its implement. International Journal of Advanced Education and Research, 2(3), 221223.

Olazabal, V. (29 de septiembre de 2016). India lanza "ataques quirúrgicos" en la frontera con Pakistán. El Mundo. Disponible en http://www. elmundo.es/internacional/2016/09 /29/57ece165e5fdea562c8b45f3. html

Oropeza, A. (2015). Ancestralidad y derecho: el sistema jurídico en la India. Revista Mundo Asia Pacífico, 4(6), 6-24. doi: 10.17230/map.v4.i6.01.

Osorno, G. (1995). El vínculo entre los ámbitos interno e internacional. De la política de eslabones a la diplomacia de doble filo. Foro Internacional, 35(3 [141]), 426-447.

Parlamento del Reino Unido. (1935). Government of India act, 1935.
Recuperado de http://www.legislation.gov.uk/ukpga/1935/2/pdfs/ ukpga_19350002_en.pdf

Pattanaik, S. (16 de mayo de 2014). Politics of Illegal Immigration and India Bangladesh Relations. Issue Brief, Institute for Defense Studies and Analyses. Recuperado de http://www.idsa.in/issuebrief/Politicsoflllegallmmigration_sspattanaik_160514

Putnam, R. (1996). Diplomacia y política nacional: la lógica de los juegos de doble nivel. En O. Gil y L. Sanz (eds.), Las fuentes internacionales de las políticas domésticas (pp. 69-120). Madrid: Zona Abierta.

Rosenau, J. (1973). Hacia el estudio de las vinculaciones nacionales-internacionales. En D. Singer y J. Rosenau, Sistema global, subsistemas y vinculaciones nacionales-internacionales. Buenos Aires: Nueva Visión.

Sahni, V. (junio-agosto, 2013). India: a pesar de sus limitaciones, una potencia emergente. Nueva Sociedad, (246), 102-114.

Sen, A. (2007). India contemporánea. Entre la modernidad y la tradición. Barcelona: Gedisa.

Thapar, R. (1989). Imagined Religious Communities? Ancient History and the Modern Search for a Hindu Identity. Modern Asian Studies, 23(2), 209-231. Recuperado de http://www.jstor.org/stable/312738

Tharoor, S. (2007). The Elephant, the Tiger and the Cellphone: Reflections 
on India, the Emerging 21st-Century Power. Nueva Delhi: Penguin.

Veer, P. van der. (1994). Religious Nationalism. Hindus and Muslims in
India. Berkeley: University of California Press. 
